\title{
Stellar matter distribution with scale-invariant hierarchical structuring
}

\begin{abstract}
In this paper we start from astronomical observations confirming the fact that cosmic matter in form of stars and galaxies, at least in more recent cosmological times, is not homogeneously, but hierarchically distributed with respect to our cosmic vantage point and typically is described by two-point correlation functions. As we show here, with these correlations also a hierarchically structured cosmic mass distribution is associated. This stellar matter distribution enables to derive a law according to which the average cosmic mass density systematically falls off with cosmic distance. At larger distances comparable with the scale $R$ of the universe also the cosmic space-time geometry hereby has to be taken into account and the results strongly depend on the curvature of the universe. We show solutions for the average mass density for positively and negatively curved $(k= \pm 1)$ and for Euclidean $(k=0)$ universes. The interesting result is that only for positively curved universes $(k=+1)$ one obtains finite values for the asymptotic mass density, while for other geometries the average mass density values monotonously fall off with cosmic distance not allowing for a reasonable input into the energy-momentum tensor $T_{i k}$ of Einstein's GTR field equations. We discuss the cosmologically essential question upcoming in this article, whether or not a positively curved universe in view of such results needs to be accepted.
\end{abstract}

Keywords: cosmic matter, stars-galaxies, structured cosmic mass distribution, curvature of the Universe, Einstein`s GTR field equations
Volume 3 Issue 4 - 2019

\author{
Hans J Fahr,' Michael Heyl² \\ 'Argelander Institut für Astronomie, Universität Bonn Auf dem \\ Hügel 7I, Germany \\ ${ }^{2}$ Deutsches Zentrum für Luft und Raumfahrt (DLR) \\ Königswinterer Str. 522 - 524, 53227 Bonn, Germany
}

Correspondence: Hans J Fahr, Argelander Institut für Astronomie, Universität Bonn Auf dem Hügel 71, 53 I2I Bonn, Germany, Email hfahr@astro.uni-bonn.de

Received: June 26, 2019 | Published: July II, 2019

\section{Introduction}

It is generally known that Einstein's general relativistic field equations (see Einstein,1915,1917, or later presented in books e.g. by Rindler, 1977, or Tolman, 1987) ${ }^{1,2}$ describe the 4-dim space time geometry through the gravitational geometry source, i.e. through the cosmic energy-momentum tensor Tik .Oppositely to what is commonly thought, this source tensor is not an easy-to-handle quantity, since the tensor ingredients are dependent on cosmic time in a non-trivial, but fairly complicated, and in a physically not evident or straightforward way. Even though all cosmological models start from the basis of the cosmological principle requiring that the universe at identical cosmic times looks the same from all space points in the universe, this does not make it evident what that means in terms of these $T_{i k}$-tensor-ingredient data, even not in such a homogeneous and isotropic universe. In case the matter content of the universe can be described as a homogeneously distributed baryonic gas, then the mass density and the scalar pressure of this gas may count as spaceaveraged quantities, but in later, closer to the present phases of the universe, when matter is structured in stars, galaxies and galaxy clusters what in replace of these former quantities should be used then? In terms of gravity sources baryons imprisoned in the body of a star are not like the same number of baryons freely distributed as a cosmic baryon gas. Stellar baryons are much hotter and in the stellar interiors their pressure may strongly count in terms of $T_{i k}$-ingredients. So the question arises how to make spatially averaged quantities out of them under such conditions.

While already this aspect makes the $T_{i k}$-ingredients a deeply problematic quantity, there is still another aspect which makes them an even much more problematic quantity. Namely the observationally confirmed fact that stellar and galactic matter is not homogeneously distributed in space but in a hierarchical structure seen in the visible light, making it an even more problematic question how under these conditions spatial averages of the $T i k$ - ingredients as they are nevertheless needed for Einstein's GRT field equations can be reasonably well constructed. In the following part of the paper we shall attempt to give an answer to this delicate question. While in earlier papers ${ }^{3-5}$ we considered the effect of gravitational binding energy formation connected with hierarchical clusterings, we here shall especially study what average cosmic mass densities under these conditions could mean.

\section{The hierarchically structured cosmic mass density}

We start from the astronomical observations carried out by Bahcall et al. ${ }^{6}$ and Bahcall et al., ${ }^{7}$ and or equally well in more recent times by Sylos-Labini et al., ${ }^{8,9}$ and take their two-point correlation function $\xi(1)$ denoting the probability to find other stellar objects at a distance $l$ from any other arbitrarily taken stellar object. The quantity $l$ has to be considered as the so-called distance parameter; astronomers take it to be identical with the visual or redshift distance, making it evident that it necessarily is a cosmologically biased quantity. From this function $\xi(1)$ we create the underlying model of cosmic matter distribution. This correlation function has been confirmed and supported by astronomical observations of the visible star and galaxy formation structure which is at present surrounding us at our cosmic standpoint, and, based on the generally respected cosmological principle, also should surround every other cosmic space point in an analogous and equivalent manner, unless the cosmological principle would turn out to be violated. This two-point correlation function $\xi(1)$ defines the probability to find another star (or galaxy) at a distance $l$ from our arbitrary standpoint and, based on astronomical observations, is expressed in the form: 


$$
\xi(1)=\xi_{0} \cdot\left(\frac{l_{0}}{l}\right)^{\alpha}
$$

where $\xi_{0}$ is a reference value valid at the reference distance $l 0$. The correlation index $\alpha$ has been determined by Bahcall et al. ${ }^{6}$ as $\alpha=1.8$. This first of all has an interesting consequence which we want to mention first here: If we may assume here that this form of a stellar or galactic clustering continues to prevail to larger and larger cosmic distances (i.e.: scale-invariant clustering!), then first of all this fact perhaps could give an evident solution of the Olbers paradox (H.W.M. Olbers, 1826), namely the fact that the sky during night is dark. Other solutions meanwhile have been offered, all perhaps worth a discussion, but none convincing up to the present, only one has been overlooked up to now. Because under these above mentioned conditions of a scale-invariant stellar clustering one would simply obtain the following growth $O\left(l_{\infty}\right)$ of the illuminated part of the sky in any arbitrary direction with a view cone $d 2 \Omega$

$$
\begin{aligned}
& O\left(l_{\infty}\right)=\frac{1}{l_{\infty}^{2} d 2 \Omega} \int_{l_{0}}^{l_{\infty}} l^{2} d^{2} \Omega . \xi(1) \frac{\pi r_{S}^{2}}{l 2} d l= \\
& \frac{1}{l_{\infty}^{2}} \int_{l 0}^{l \infty} l 2 . \xi(1) \frac{\pi r_{S}^{2}}{l 2} d l=\frac{\pi r_{S}^{2}}{l_{0}^{2}} \xi_{0} \cdot l_{0}^{\alpha} \int_{l 0}^{l \infty} \frac{1}{l \infty} d l= \\
& \pi r_{S}^{2} \xi_{0} l_{0}^{\alpha-2} \frac{l_{0}^{\alpha-2}}{1-\alpha}\left[\left(\frac{l \infty}{l 0}\right)^{1-\alpha}-1\right]
\end{aligned}
$$

First here one can see that for $\alpha=0$ (i.e. no structuring; homogeneous matter distribution!) one would have the following result

$$
O\left(l_{\infty}\right)=\pi r_{s}^{2} \xi_{0} l_{0}^{-1}\left[\left(\frac{l \infty}{l 0}\right)-1\right]
$$

clearly showing that for increasing values of $(l \infty / l 0)$ the sky coverage would grow to $O\left(l_{\infty}\right)>1$ (i.e. illuminated sky $=$ Olbers paradox!).

To the contrast, however, for values as observationally confirmed, namely alpha $=1.8$, one can see that $O\left(l_{\infty}\right)$ always leads to values $O\left(l_{\infty}\right)>1$ (i.e. non-illuminated sky $=$ no Olbers paradox!).

To continue now with another interesting problem connected with the above mentioned clustering we here want to emphasize the cosmologically important point, that the existence of the above correlation function can evidently also be interpreted as an expression for the structured stellar density or stellar mass density distribution of surrounding stars or galaxies in our cosmic environment, and consequently thereby also expresses a standpoint-oriented mass density distribution $p=p(l)$ when recognizing that the mass is closely associated with the number of stars with a typical stellar or galactic mass $m_{0}$ on a spherical shell at distance $l$, based at a first approximation here, Euklidean geometry conditions (i.e. flat universe: $k=0$ ), is then given by

$$
d M(l)=4 \pi l 2 m_{0} \xi_{0}\left(\frac{l 0}{l}\right)^{\alpha} d l=4 \pi \rho 2 \cdot\left(\frac{l 0}{l}\right)^{\alpha} d l
$$

with $\rho_{0}$ denoting a reference value of the hierarchy-typical mass density. Bahcall ${ }^{7}$ and Chokski $^{7}$ point out furthermore the astonishing fact that the general type of the above mentioned two-point correlation function $\xi(1)$ interestingly enough is observationally confirmed as well for galaxy correlations, as for cluster correlations, as also for super-cluster correlations, with the difference that only the reference scale $l 0$ and the reference probability $\xi_{0}$ or mass density $\rho_{0}$ have to be adapted from the galaxy-case up to the super-cluster case, while however as a surprise the same correlation index of $\alpha=1.8$ is reappearing as a common number for all these hierarchies.

Taking the largest hierarchy, i.e. super-clusters, in order to cover the largest achievable distances of the order $100 \mathrm{Mpc}$ and more, we thus would use the corresponding SC-SC correlation function, and the corresponding mass increment with distance $l$ would then - in accordance with Eq.(4) - be given by

$$
\begin{aligned}
& d M(l)=4 \pi l 2 m_{S C, 0} \xi_{S C, 0} \cdot\left(\frac{l S C, 0}{l}\right)^{\alpha} d l= \\
& 4 \pi l^{2} \rho_{S C}, 0 \cdot\left(\frac{l S C, 0}{l}\right)^{\alpha} d l=
\end{aligned}
$$

where $\rho_{S C}, 0$ is a typical reference density for the super cluster scale $l_{S C}, 0$. In order to address the largest achievable cosmic distances $l \simeq R, R$ being the scale of the universe, where the prevailing cosmic geometry conditions become important, we should then also take furthermore into account that most likely we are sitting in a non-Euklidean universe with a curved general-relativistic pace time geometry. One therefore would have to pay attention to the fact that the radial distance parameter $l$, in the Robertson-Walker approximation of the cosmic geometry, is transformed into a geometrical distance $r(l)$ given by (see e.g. Goenner ${ }^{10}$, or Fliessbach ${ }^{11}$ ):

$$
r(l)=l \cdot(1+k l 2)^{-1}
$$

i.e. the spherical area associated to the distance parameter $l$ is $\Phi(1)=4 \pi 2(l)$ where $k$ denotes the cosmic curvature parameter which needs to be determined by looking for the best fitting cosmological FLRW-model (e.g. see Bennet et al. ${ }^{12}$ yielding $k \simeq 0$ ).

This latter cosmological model interestingly enough needs, however, spatially averaged quantities as $T_{i k}$-ingredients which latter, under the perspectives given above, are highly problematic quantities. Anticipating the value for $k$ as input from this model one can include this geometric distance transformation and would bring the above Eq. (5) for $d M(l)$ into the following form:

$$
d M(l)=4 \pi l 2+.(1+k l 2)^{-2} \cdot \rho_{S C}, 0 \cdot\left(\frac{l S C, 0}{l}\right)^{\alpha} \cdot \frac{(1-k l 2)}{(1+k l 2)^{2}} d
$$

or rearranging things and putting them into a better logic order then gives

$$
d M(l)=4 \pi \rho_{S C}, 0 .\left(\frac{l S C, 0}{l}\right)^{\alpha} \cdot \frac{(1-k l 2)}{(1+k l 2)^{4}} l 2 d l
$$

The cosmic curvature parameter $k$ can be restricted to values of $k=0 ; k= \pm 1$ if $l$ is scaled with the cosmic scale parameter $R=R(t)$ by $k=K . R 2, K$ being the cosmic curvature scalar or the contracted Ricci tensor $K=R_{i}^{i}$ (i.e. Ricci scalar). Therefore, 
besides the Euklidean case $k=0$, favoured by Bennet et al. ${ }^{12}$ one obtains the following two more options:

$$
\begin{aligned}
& d M(l)=4 \pi \rho_{S C}, 0 l_{S C, o}^{\alpha} \cdot \frac{\left(1 \mp \frac{l 2}{R 2}\right)}{\left(1 \pm \frac{l 2}{R 2}\right)^{4}} l 2-\alpha d l= \\
& 4 \pi \rho_{S C}, 0 l_{S C, o}^{\alpha} \cdot g(l) d l
\end{aligned}
$$

\section{The average cosmic mass density}

Considering here the case of $k= \pm 1$ (as the most promising with respect to asymptotically reach a constant mass density $\rho_{\infty}=\rho(l \rightarrow \infty)$ with the over-Euklidean growth of the sphere surface at large distances $l \simeq R$ leads to the following remaining geometrical expression: ${ }^{13}$

$$
g(l) d l\left[\frac{\left(1+\frac{l 2}{R 2}\right)}{\left(1-\frac{l 2}{R 2}\right)^{4}} l 2-\alpha d l\right]
$$

which at large distances $l \simeq R$ leads to the following asymptotic behaviour

$$
g(l \rightarrow R)=\lim _{l \rightarrow R}\left[\frac{\left(1+\frac{l 2}{R 2}\right)}{\left(1-\frac{l 2}{R 2}\right)^{4}} l 2-\alpha d l\right]=\frac{2}{1-\delta} l 2-\alpha d l
$$

For small distances $(l / R) \ll 1$ one would instead obtain the following behaviour

$$
\begin{aligned}
& g(l \ll R)=\left[\frac{\left(1+\frac{l 2}{R 2}\right)}{\left(1-\frac{l 2}{R 2}\right)^{4}} l 2-\alpha d l\right] l \ll R= \\
& \left(1+\frac{l 2}{R 2}\right)\left(1+4 \frac{l 2}{R 2}\right) l 2-\alpha d l \simeq\left(1+5 \frac{l 2}{R 2}\right) l 2-\alpha d l
\end{aligned}
$$

After this inspection one can then state that the expression for the average cosmic density in such a hierarchically structured universe for example with $k=-1$ finally takes the following form:

$$
\rho(l)=\frac{M(l)}{V(l)}=m_{S C}, 0 \xi_{S C}, 0 l_{S C, 0}^{\alpha} \frac{\int_{0}^{1}\left[\frac{\left(1+\frac{l 2}{R 2}\right)}{\left(1-\frac{l 2}{R 2}\right)^{4}} l 2-\alpha d l\right]}{\left(1+\frac{l 2}{R 2}\right)} \int_{0}^{1}\left[\frac{\left(\frac{l 2}{R 2}\right)^{4}}{\left(1-\frac{1}{R 2} d l\right]}=\right.
$$

In the presented Figures $1,2,3$ we show the quantities $M(l), V$ $(l)$ and $\rho(l)$ as functions of $l$, for $k=+1, k=0$ and $k=-1$, respectively. ${ }^{14}$

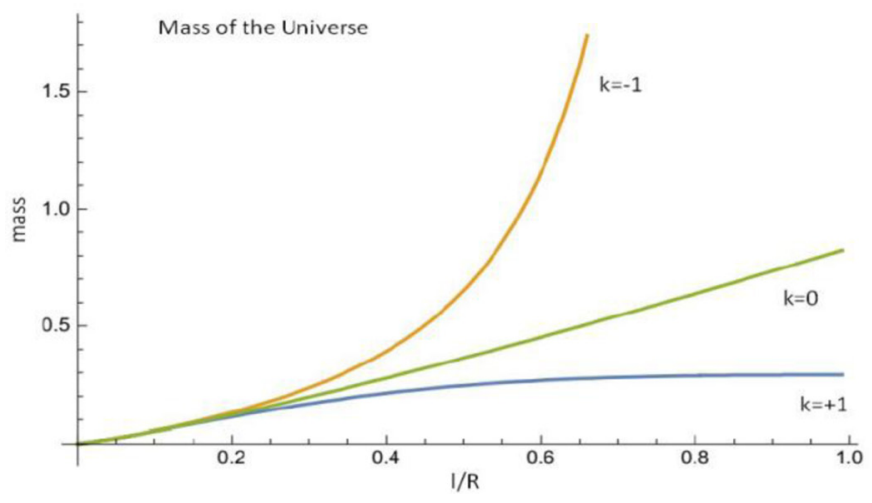

Figure I This shows the increase of the cosmic mass as a function of $I$ in arbitrary units for curvature $k=-1, k=0$ and $k=1$, respectively.

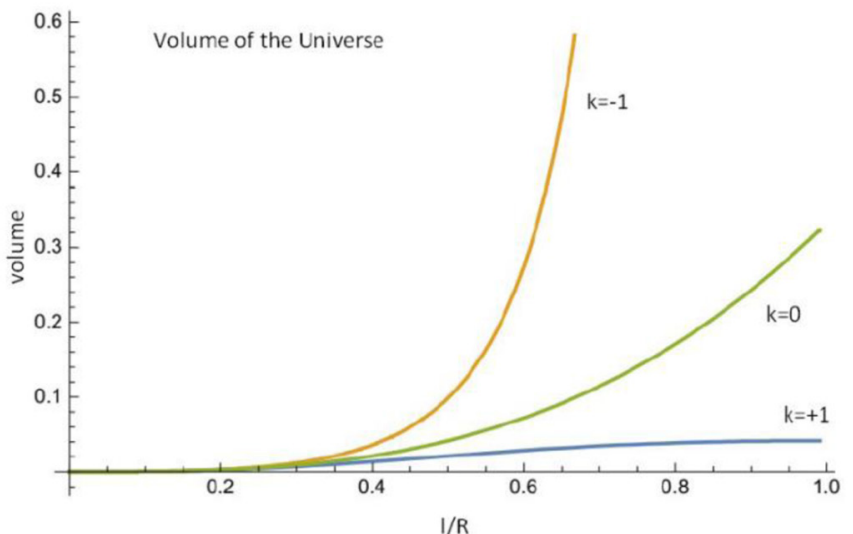

Figure 2 This shows the increase of the cosmic volume as a function of $I$ in arbitrary units for curvature $k=-1, k=0$ and $k=1$, respectively.

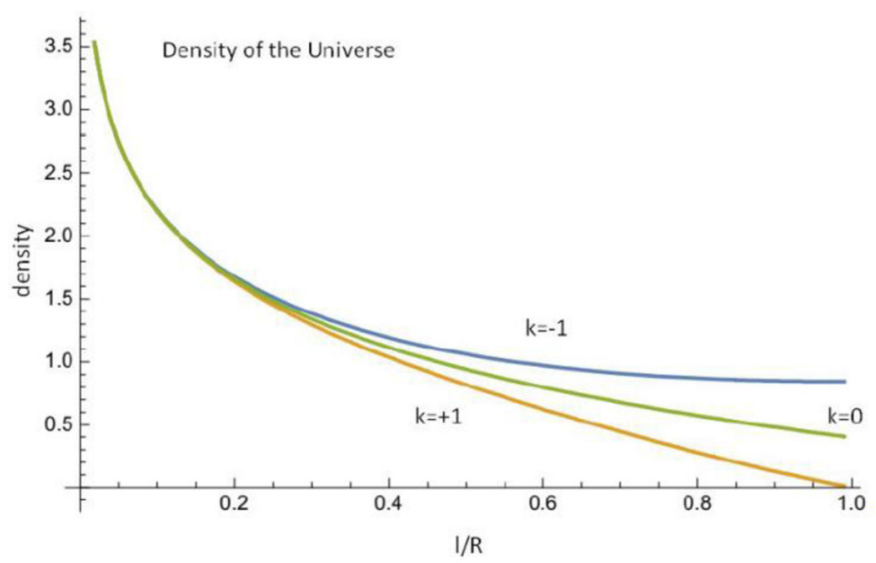

Figure 3 This shows the increase of the cosmic density as a function of $I$ in arbitrary units for curvature $K=+1, k=0$ and $\mathrm{k}=-\mathrm{I}$, respectively.

\section{Obtained results}

In the preceding section of this paper we have taken serious the astronomically confirmed fact that we are surrounded by a hierarchically structured stellar universe. In order to be cosmologically based on this important fact, we have first derived a point-related spatial mass configuration of surrounding stellar mass sources from the astonomically confirmed two-point correlation functions $\xi(l)$ 
describing the spatial configuration of radiating stellar or galactic sources. As we have derived in this paper there exists the following connection between this correlation function and a hierarchy typical mass density

$$
0=m_{0} \xi_{0}
$$

where $m_{0}$ denotes the average mass of the hierarchy-typical source, i.e. either stars, or galaxies, or clusters of galaxies. For the spatially largest, observed hierarchies, super clusters, this would give a mass density typical for super clusters given by

$$
\rho_{S C, 0}=m_{S C, 0} \xi_{S C, 0}
$$

Since astronomers (Bahcall, ${ }^{6}$ Bahcall ${ }^{7}$ and Chokski ${ }^{7}$ ) have given there correlation function in absolute numbers, one thus from their results can determine absolute values of hierarchy-typical mass densities, e.g. like the value $\rho_{S C, 0}$ Figure1 \& Figure 2.

Studying therefore our hierarchical density distributions in Figure 3 we find that only for the case of a positively curved universe, i.e. $k=+1$, a constant value for the cosmic density at $l \rightarrow R$ can be expected, namely just the value $\rho_{S C, 0}$.In contrast to that result, for Euclidean and negatively curved universes, i.e. $k=0$ or $k=-1$, no finite density is to be expected, but density values that permanently decrease with increasing values of $l$. But now one has to clearly see the critical point from that outcome - namely that without a finite density value for the density of the universe, one cannot enter and solve the FLRW-field equations.

\section{Conclusion}

In the frame of our deductions only one cosmic solution can be envisioned - the one with a positively curved universe and a finite cosmic density of $\rho=\rho_{S C, 0}$. But in order to logically and scientifically bring things together one has to make sure that a universe with this density $\rho_{S C, 0}$ is a positively curved one. In order to be in fact positively curved $(k=+1)$, the density of such a universe has to be larger than it scritical density

$$
\rho_{c}=3 H_{0}^{2} / 8 \pi G \leq \rho_{S C, 0}
$$

In order to decide upon that, one needs to know the Hubble constant $\mathrm{Ho}$ of the present universe and the density value $\rho_{S C, 0}$

. At this point we have to recognize a special problem for the continuation of the above presented chain of ideas, namely the question concerning the actual value of the Hubble constant $H o$ which is extremely essential for our above ideas. The most modern value of this constant, $H o=73 \mathrm{~km} / \mathrm{s} / M_{p c}$, is derived with the help of the standard_CDM- cosmological model on the basis of a Robertson-Walker spacetime geometry, trying to fit into the cosmological expansion model the cosmic matter evolution from the times of matter recombination (Bennet et al., 2003) to the present times of visible galactic structurings (millennium simulation!, see Springel et al., 2005). The essential finding from these attempts is that the energetic ingredients of this best-fitting universe are: $\Omega \wedge=0.72 ; \Omega D=0.23 ; \Omega B=0.04 ; \Omega \vee=0$ !, where the different $\Omega$ values are defined by

$$
\Omega_{i}=\frac{\rho i}{\rho c}=\frac{\rho i}{\left(\frac{3 H_{0}^{2}}{8 \pi G}\right)}
$$

with ${ }_{i}=\wedge ; \mathrm{i}=\mathrm{D} ; \mathrm{i}=\mathrm{B}$ determining the critical density of the vacuum, of the dark matter, and of the baryonic matter, respectively, and $\rho_{c}$ denoting the so-called critical cosmic density, critical with respect to the actually prevailing curvature of the universe, and with the validity:

$$
\Omega \wedge+\Omega D+\Omega B+\Omega \vee+\Omega k=1
$$

Hereby the cosmic curvature energy $\Omega k$ is defined by

$$
\Omega k=-\frac{1}{H_{0}^{2}} \frac{k c 2}{R_{0}^{2}}=-k \frac{c 2}{R_{0}^{2}}
$$

and, in the above mentioned simulation model, with $k=0$, has been set to $\Omega_{k}=0$ ! Also the contribution of cosmic photons for the cosmic times after recombination has been neglected setting $\Omega_{\vee}=0$ ! . If on the other hand, however, one would have to admit a positive curvature with $k=+1$, as e.g. favoured in the argumentation of the upper part of this paper, one would obtain a new cosmic energy balance given by ( see e.g. Goenner, 1996, Fahr, 2017):

$$
\Omega \wedge+\Omega D+\Omega B+\Omega k=\Omega \wedge+\Omega D+\Omega B-\frac{c 2}{R_{0}^{2}}=1
$$

With the values $\Omega i$ found in the millennium fit (Springel et al., $2005)$ the above requirement evidently would not be fulfilled. Instead one would have to enhance the sum $\sum \Omega i$ of the present values $\Omega i$ by an amount of $(c / \dot{R} 0)^{2}$, which by the way could be achieved, when keeping the relative contributions of the $\Omega i$-terms, by lowering the value of the actual Hubble constant $H 0$. This means that , admitting a positive (instead of a vanishing) curvature $k$, one would either need a completely new fit to the cosmic data, evidently also leading to a new value of the best-fitting Hubble constant $H 0$, or perhaps try the following way: One could perhaps argue that asymptotically (e.g. for $\Omega \wedge \rightarrow 1$ ) the $\wedge C D M$-universe will approach an expansion rate with a "constant" Hubble constant $\mathrm{H} \wedge$ given by

$$
\mathrm{H}_{\wedge}^{2}=\frac{8 \pi G \rho \wedge}{3}=\mathrm{H}_{0}^{2} \Omega \wedge 0=0.72 . \mathrm{H}_{0}^{2}
$$

meaning that the asymptotic Hubble constant would be smaller than the presently determined Hubble constant by the factor $\sqrt{0.72}$, i.e. yielding $\mathrm{H} \wedge=0.85 H_{0}=62,5 . \mathrm{km} / \mathrm{s} / M_{p c}$.

\section{Acknowledgements}

None.

\section{Conflicts of interest}

The author declares there is no conflict of interest.

\section{References}

1. Rindler W. Essential Relativity: Special, General, and Cosmological. $2^{\text {nd }}$ edn, Springer-Verlag, New York. 1977.

2. Tolman RC. Relativity, Thermodynamics, and Cosmology. Dover Publications, New York, 1987.

3. Fahr HJ, Heyl M. About universes with scale $\square$ related total masses and their abolition of presently outstanding cosmological problems. Astron Notes. 2007;328(2):192-198.

4. Fahr HJ, Sokaliwska M. In Innovative Cosmology; Aspects of Todays Cosmology. $2^{\text {nd }}$ In: A Alfonso-Faus, editor. ISBN 978-953-307-626-3. 2011. p. $95-120$. 
5. Fahr HJ, Sokaliwska M. The influence of gravitational binding energy on cosmic expansion dynamics, new perspectives for cosmology. Astrophys Space Sci. 2012;339(2):379-387.

6. Bahcall NA. Large scale structure in the universe indicated by galaxy clusters. Ann Rev Astron Astrophys. 1988;26:631-686.

7. Bahcall NA, Chokski A. The clustering of radiogalaxies. Astrophysical Journal Part 2 -Letters. 1992;385:L33-L36.

8. Sylos-Labini F. Gravitational fluctuations of the galaxy distribution. Astron Astrophys. 2010;523:A68.

9. Sylos-Labini F, Vasilyev NL. Extension and estimation of correlations in cold dark matter models. Astron Astrophys. 2008;477(2):381-395.
10. Goenner H. Einführung in die Spezielle und Allgemeine Reltivitätstheorie, Spektrum Akad. Verlag, Heidelberg-Berlin-Oxford. 1996.

11. Fliessbach T. Allgemeine Relativitätstheorie, Wissenschaftsverlag BI, Mannheim/Wien/Zürich. 1990.

12. Bennet CL. Hill RS, Hinshaw G, et al. First-Year Wilkinson Microwave Anisotropy Probe (WMAP) Observations: Foreground Emission. Astrophys J Suppl Ser. 2003;148(1):97-117.

13. Einstein A. Die Feldgleichungen der Gravitation. Sitzungsber d Preuss Akad Wissensch. 1915;48:844-847.

14. Einstein A. Kosmologische Betrachtungen der allgemeinen Relativitätstheorie. Sitzungsber Preuss Akademie Wissenschaft. 1917;55:142-152. 